\title{
Association between a Polymorphism in miR-34b/c and Susceptibility to Cancer - a Meta-analysis
}

\author{
Zhuo Lin ${ }^{1}$, Li Chen ${ }^{2}$, Mei Song ${ }^{1}$, Ke-Qing Shi ${ }^{1}$, Kai-Fu Tang ${ }^{2 *}$
}

\begin{abstract}
MicroRNAs (miRNAs) act as tumor suppressors or promoters in neoplasia by regulating relative geneexpression. The association between a single nucleotide polymorphism (SNP) rs4938723 in miR-34b/c and susceptibility to cancers was inconsistent in previous studies. In this study, we conducted a literature search of PubMed, Web of Science and Embase to identify all relevant studies in this meta-analysis with 6,036 cases and 6,204 controls. We found that the miR-34b/c rs4938723 polymorphism was significantly associated with increased risk of cancers in the heterozygous model (TC versus TT, $\mathrm{OR}=1.09,95 \% \mathrm{CI}=1.01-1.18, P=0.02)$. Subgroup analysis also revealed increased risk for Asian ethnicity in the heterozygous model (TC versus TT, OR=1.12,95\% CI=1.02-1.22, $P=\mathbf{0 . 0 2}$ ), but decreased risk of colorectal cancer in homozygote model (CC versus TT, $O R=0.66$, 95\% $\mathrm{CI}=0.47-0.92, P=0.02)$ and in the recessive model $(\mathrm{CC}$ versus $\mathrm{TC}+\mathrm{TT}, \mathrm{OR}=\mathbf{0 . 6 7}, 95 \% \mathrm{CI}=\mathbf{0 . 4 8 - 0 . 9 3 , P = 0 . 0 2 )}$ by cancer type. The current meta-analysis indicated that the miR-34b/c rs4938723 polymorphism may decrease susceptibility to colorectal cancer. Well-designed studies with larger sample size are required to further validate the results.
\end{abstract}

Keywords: miR - 34b/c - rs4938723 - polymorphism - cancer - meta-analysis

Asian Pac J Cancer Prev, 15 (17), 7251-7255

\section{Introduction}

MicroRNAs (miRNAs) are a class of non-coding RNA of approximately 20-24 nucleotides in length and highly evolutionarily conserved, which act the role of significant regulators in post transcription of gene expression. Emerging evidence indicates that miRNAs are related with various biological processes, such as cell proliferation, apoptosis, differentiation and metabolism, particularly in carcinogenesis that act as regulators to regulate the expression of tumor genes (Ambros, 2004; Jansson et al., 2012; Ji et al., 2014). Although the exact mechanism underlying miRNA deregulation in cancers remains unknown, some key dysregulated miRNAs have already been used as molecular biomarkers, which could improve diagnosis, prognosis, and monitoring of treatment response for cancers (Ruan et al., 2009). Single nucleotide polymorphisms (SNPs) are a kind of genetic variations associated with population diversity, disease susceptibility, drug metabolism and genome evolution (Hanchard, 2005). SNPs located in miRNA genes region could affect miRNA expression or maturation and then contribute to cancer risk (Parlayan et al., 2014). Thus, SNPs in miRNAs are ideal predictive factor for cancer risk, because they could generate significant effect in the development of cancers.
As some miRNAs appear to have their own promoters, SNPs in the promoter region may play critical roles in the development of human cancers. Recently, a potentially functional polymorphism rs4938723 (T>C) has been discovered in the promoter region of $\mathrm{miR}-34 \mathrm{~b} / \mathrm{c}$. The $\mathrm{T}$ to C shift of the rs4938723 polymorphism was predicted to influence the GATA-X binding sites. If the polymorphic location is $\mathrm{C}$, it can bind to the GATA-X transcription factors; otherwise, it cannot bind to the GATA-X transcription factors. GATA-X transcription factors can activate or repress expression of target genes when they bind to a specific DNA sequence (A/T) GATA (A/G) in the region of promoters (Bossard et al., 1998). In recent years, some case-control studies have been conducted to determine the association between rs 4938723 and multiple kinds of cancers in various types, such as hepatocellular carcinoma (Xu et al., 2011; Han et al., 2013; Son et al., 2013), colorectal cancer (Gao et al., 2013; Oh et al., 2014), renal cell cancer (Zhang et al., 2014), esophageal cancer (Yin et al., 2013), nasopharyngeal carcinoma (Li et al., 2013) and breast cancer (Bensen et al., 2013). However, the results are conflicting and inconclusive. Therefore, we conducted the present meta-analysis of all relevant studies from publication to obtain a more accurate evaluation of the association between miR-34b/c rs4938723 polymorphism and cancer risk.

${ }^{I}$ Department of Infection and Liver Diseases, Institution of Hepatology, the First Affiliated Hospital of Wenzhou Medical University, ${ }^{2}$ Institute of Genomic Medicine, Wenzhou Medical University, Wenzhou, China *For correspondence: tang_kaifu@aliyun.com 


\section{Materials and Methods}

\section{Search Strategy and Study Selection}

We conducted a comprehensive search on PubMed, Web of science and Embase to identify all potentially eligible studies on miR-34b/c rs4938723 polymorphism and cancer risk. The search covered all papers published up to April 10, 2014, by using the following strategy: (pre-mir-34b/c OR pri-miR-34b/c OR mir-34b/c OR microRNA-34b/c OR rs4938723) and (gene OR polymorphism OR allele OR variation) and (cancer OR carcinoma OR tumor OR tumour), without any restriction on language. References of the relevant articles on miR-34b/c polymorphism were also filtrated. Eligible studies should conform to all of the following criteria: 1) estimation of miR-34b/c rs2938723 polymorphism and cancer risk; 2) independent case-control studies; 3 ) sufficient data for estimating the odds ratio (OR) with 95\% confidence interval $(\mathrm{CI})$ and a $P$-value. If one study included two or more case-control studies by subgroup such as ethnicity, then it would be divided into two or more independent studies.

\section{Data Extraction}

Serviceable informations were extracted independently by two investigators from all eligible studies accorded with the inclusion criteria. Disagreements were reconciled through discussion between the two investigators. The following informations were extracted from each study: first author's name, publication year, origin country, ethnicity of study population, cancer type, genotyping method, numbers of cases and controls for each genotype. Genetic equilibrium of miR-34b/c polymorphism under Hardy-Weinberg equilibrium (HWE) was evaluated by using the chi-square test for each study in controls (Wigginton et al., 2005).

\section{Statistical Analysis}

Crude OR with 95\% CI was applied to evaluate the strength of the association of miR-34b/c rs4938723 polymorphism and cancer risk (Woolf, 1955). The statistical significance of the pooled OR was detected by Z-test and $p<0.05$ was considered statistically significant. We investigated the association between genetic variants and cancer risk in allele model ( $\mathrm{C}$ versus $\mathrm{T}$ ), homozygous model (CC versus TT), heterozygous model (TC versus TT), dominant model (CC+TC versus TT) and recessive model (CC versus TC+TT), respectively. Subgroup analysis was also carried out by ethnicity and cancer type (if one ethnicity or one cancer type contained only one single study, it was merged into other subgroups).

The statistical heterogeneity between studies was detected by Q-test (Higgins et al., 2003) and a $p$-value of $<0.05$ or I 2 value of $\geq 50 \%$ was considered to be representative of statistically significant heterogeneity, then the pooled OR was calculated by the random-effects model (DerSimonian et al., 2007). Otherwise, the fixedeffects model was used in meta-analysis (Mantel et al., 1959). Sensitivity analysis was performed to assess the stability of the results. Each study was removed in turn from the total, and the remaining studies were reanalyzed (Thakkinstian et al., 2005). Publication bias was detected by Begg's funnel plot and Egger's linear regression method with $p<0.05$ being considered statistically significant (Egger et al., 1997).

All statistical analysis was carried out using the the STATA software package version 12.0 (STATA Corp, College Station, Texas) and $p<0.05$ was considered statistically significant.

\section{Results}

\section{Study Identification}

In accordance with the inclusion criteria, 10 eligible studies were collected in this meta-analysis, with 6036 cases and 6204 controls. The details of the selection process were presented in Figure 1. Among the 10 studies, 8 studies were conducted in Asian population, 1 study was conducted in Caucasian population, and 1 study was conducted in African population. Various cancer types included hepatocellular carcinoma (3 studies), colorectal cancer (2 studies), breast cancer $(2$

Table 1. Main characteristics of studies included in the meta-analysis for miR-34b/c rs4938723

\begin{tabular}{|c|c|c|c|c|c|c|c|c|c|c|c|c|c|}
\hline \multirow[t]{2}{*}{$\overline{\text { Author }}$} & \multirow[t]{2}{*}{ Year } & \multirow[t]{2}{*}{ Country } & \multirow[t]{2}{*}{ Ethnicity } & \multirow[t]{2}{*}{$\begin{array}{c}\text { Cancer } \\
\text { type }\end{array}$} & \multirow[t]{2}{*}{$\begin{array}{l}\text { Genotyping } \\
\text { methods }\end{array}$} & \multirow[t]{2}{*}{$\begin{array}{c}\text { Number of } \\
\text { cases/controls }\end{array}$} & \multicolumn{3}{|c|}{$\begin{array}{c}\text { Genotypes } \\
\text { distribution } \\
\text { of cases }\end{array}$} & \multicolumn{3}{|c|}{$\begin{array}{l}\text { Genotypes } \\
\text { distribution } \\
\text { of controls }\end{array}$} & \multirow[t]{2}{*}{ HWE(P) } \\
\hline & & & & & & & $\mathrm{TT}$ & $\mathrm{TC}$ & $\mathrm{CC}$ & $\mathrm{TT}$ & $\mathrm{TC}$ & $\mathrm{CC}$ & \\
\hline Zhang & 2014 & C & & $\mathrm{RCC}$ & Taq & & 302 & 324 & 84 & 352 & 344 & 64 & 2 \\
\hline $\mathrm{Oh}$ & 2014 & & & CRC & PCR-RFLP & & 272 & 233 & 40 & 216 & 171 & 41 & \\
\hline Yin & 2013 & China & Asian & ESCC & LDR & $600 / 673$ & 277 & 278 & 45 & 310 & 290 & 73 & 66 \\
\hline Son & 2013 & Korea & Asian & $\mathrm{HCC}$ & PCR-RFLP & $157 / 201$ & 69 & 75 & 13 & 110 & 74 & 17 & 0.37 \\
\hline $\mathrm{Li}$ & 2013 & China & Asian & NPC & PCR-RFLP & $217 / 360$ & 82 & 104 & 31 & 168 & 155 & 37 & 0.89 \\
\hline Han & 2013 & China & Asian & $\mathrm{HCC}$ & TaqMan & $1013 / 999$ & 451 & 444 & 118 & 456 & 424 & 119 & 0.18 \\
\hline Gao & 2013 & China & Asian & CRC & PCR-RFLP & $347 / 488$ & 175 & 144 & 28 & 216 & 210 & 62 & 0.33 \\
\hline Bensen & 2013 & America & Caucasian & $\mathrm{BC}$ & $\begin{array}{c}\text { Genotyping } \\
\text { Array }\end{array}$ & $1203 / 1088$ & 496 & 563 & 144 & 430 & 503 & 155 & 0.69 \\
\hline Bensen* & 2013 & America & African & $\mathrm{BC}$ & $\begin{array}{c}\text { Genotyping } \\
\text { Array }\end{array}$ & $742 / 658$ & 362 & 317 & 63 & 343 & 257 & 58 & 0.32 \\
\hline $\mathrm{Xu}$ & 2011 & China & Asian & $\mathrm{HCC}$ & PCR-RFLP & $502 / 549$ & 204 & 236 & 62 & 266 & 229 & 54 & 0.65 \\
\hline
\end{tabular}

RCC: renal cell cancer; CRC: colorectal cancer; ESCC: esophageal cancer; HCC: hepatocellular caicinoma; NPC: nasopharyngeal carcinoma; BC: breast cancer; PCRRFLP: polymerase chain reaction-restriction fragment length polymorphism; LDR: ligation detection reaction; HWE: Hardy-Weinberg equilibrium; P: $p$ value.* The study from the same article 
studies), and other cancers ( 3 studies). The publication years of the included articles ranged from 2011 to 2014. In addition, 5 studies used polymerase chain reactionrestriction fragment length polymorphism (PCR-RFLP), 2 studies used TaqMan assay, 2 studies used Genotyping Array, and 1 study used ligation detection reaction (LDR) for genotyping. Furthermore, genotype distribution of controls in all studies was consistent with HWE (Table 1).

\section{Quantitative Data Synthesis}

The association of miR-34b/c rs4938723 polymorphism and cancer risk was investigated in 10 studies. And results of pooled analysis revealed that an increased risk was observed for the comparison of heterozygous model (TC versus $\mathrm{TT}, \mathrm{OR}=1.09,95 \% \mathrm{CI}=1.01-1.18, P=0.02)$. In the stratified analysis by ethnicity, an association between

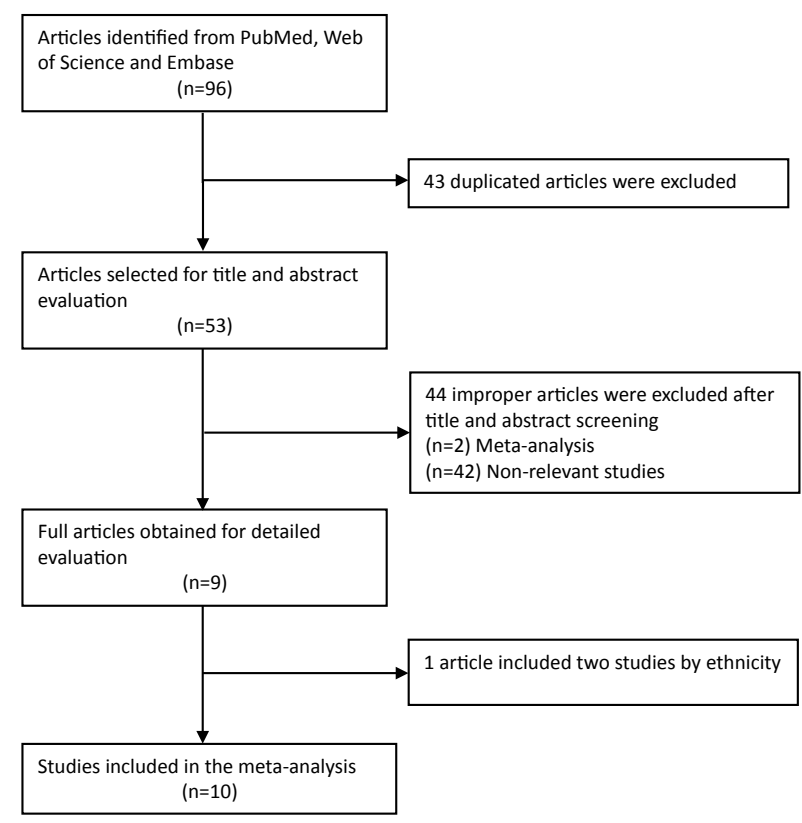

Figure 1. Flow Chart of The Study Selection Process
miR-34b/c polymorphism and cancer risk was detected in Asian population for comparison of heterozygous model (TC versus TT, OR=1.12, 95\% $\mathrm{CI}=1.02-1.22$, $P=0.02)$. Further subgroup analysis by cancer type

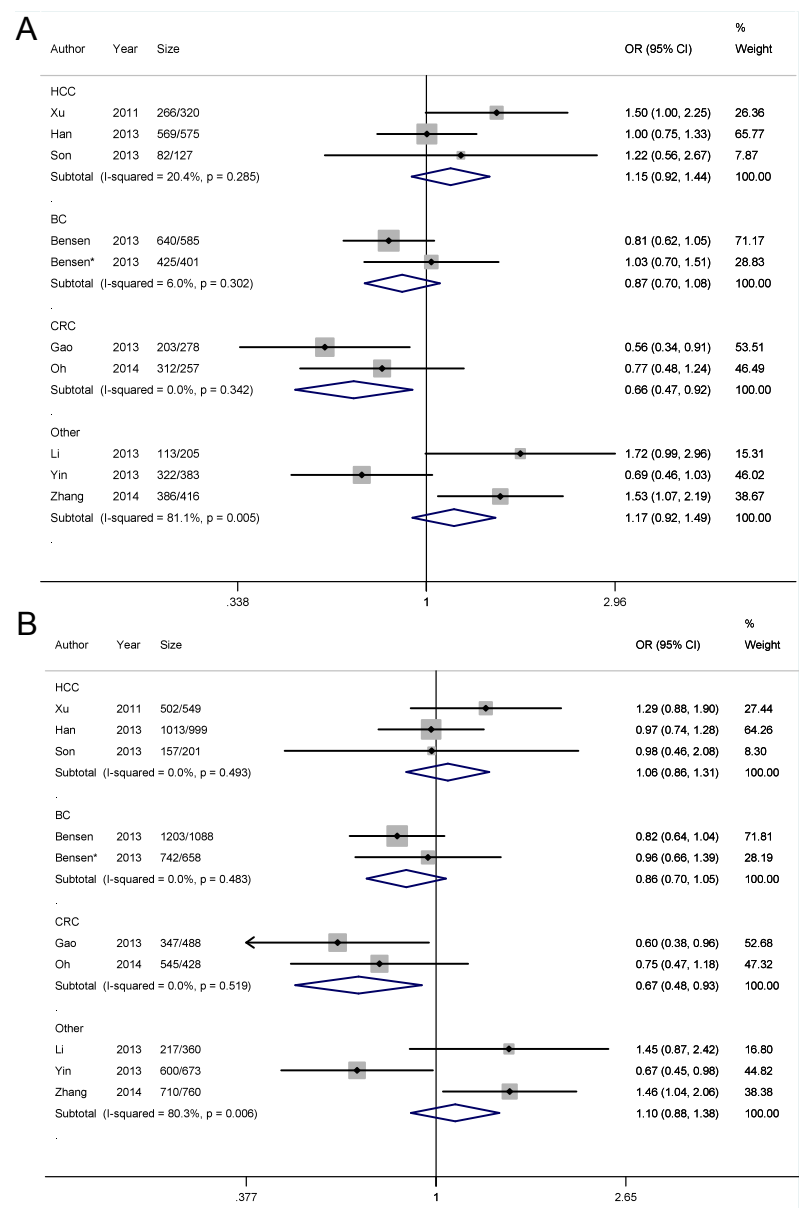

Figure 2. Forest Plot of ORs for The Association of miR-34b/c rs4938723 with The Risk of Cancers in Subgroup Analysis by Cancer Type. (A) homozygote model; (B) recessive model

Table2. Meta-analysis of miR-34b/c rs4938723 polymorphism with cancer risk

\begin{tabular}{|c|c|c|c|c|c|c|c|c|c|c|c|c|c|}
\hline \multirow[t]{2}{*}{ Studies } & \multirow[t]{2}{*}{$\mathrm{N}^{\mathrm{a}}$} & \multicolumn{4}{|c|}{ C vs. T } & \multicolumn{4}{|c|}{ CC vs. TT } & \multicolumn{4}{|c|}{ TC vs. TT } \\
\hline & & OR $(95 \% \mathrm{CI})$ & $\mathrm{P}$ & $\mathrm{P}-\mathrm{h}$ & $\mathrm{I}^{2}$ & OR $(95 \% \mathrm{CI})$ & $\mathrm{P}$ & P-h & $\mathrm{I}^{2}$ & OR $(95 \%$ CI $)$ & $\mathrm{P}$ & P-h & $\mathrm{I}^{2}$ \\
\hline Totla & 10 & $1.04(0.95,1.15)$ & 0.38 & 0.002 & 65.8 & $1.00(0.81,1.25)$ & 0.97 & 0.002 & 64.8 & $1.09(1.01,1.18)$ & 0.02 & 0.20 & 26.5 \\
\hline \multicolumn{14}{|l|}{ Cancer type } \\
\hline $\mathrm{HCC}$ & 3 & $1.15(0.97,1.36)$ & 0.10 & 0.11 & 54.2 & $1.15(0.92,1.44)$ & 0.22 & 0.29 & 20.4 & $1.25(0.99,1.57)$ & 0.06 & 0.12 & 52.6 \\
\hline $\mathrm{BC}$ & 2 & $0.98(0.84,1.15)$ & 0.84 & 0.12 & 57.6 & $0.87(0.70,1.08)$ & 0.21 & 0.30 & 6.0 & $1.04(0.91,1.20)$ & 0.55 & 0.20 & 39.8 \\
\hline $\mathrm{CRC}$ & 2 & $0.87(0.71,1.07)$ & 0.18 & 0.15 & 50.9 & $0.66(0.47,0.92)$ & 0.02 & 0.34 & 0.0 & $0.97(0.80,1.18)$ & 0.74 & 0.22 & 32.9 \\
\hline Other & 3 & $1.12(0.91,1.37)$ & 0.29 & 0.03 & 72.0 & $1.21(0.68,2.13)$ & 0.52 & 0.005 & 81.1 & $1.13(0.98,1.30)$ & 0.10 & 0.50 & 0 \\
\hline \multicolumn{14}{|l|}{ Ethnicity } \\
\hline Asian & 8 & $1.06(0.94,1.20)$ & 0.32 & 0.003 & 68.2 & $1.04(0.79,1.37)$ & 0.78 & 0.002 & 69.1 & $1.12(1.02,1.22)$ & 0.02 & 0.19 & 29.6 \\
\hline Other & 2 & $0.98(0.84,1.15)$ & 0.84 & 0.12 & 57.6 & $0.87(0.70,1.08)$ & 0.21 & 0.30 & 6.0 & $1.04(0.91,1.20)$ & 0.55 & 0.20 & 39.8 \\
\hline \multirow[t]{2}{*}{ Studies } & $\mathrm{N}^{\mathrm{a}}$ & \multicolumn{4}{|c|}{$\mathrm{CC}+\mathrm{TC}$ vs. TT } & \multicolumn{4}{|c|}{$\mathrm{CC}$ vs. TC+TT } & & & & \\
\hline & & OR $(95 \%$ CI $)$ & $\mathrm{P}$ & P-h & $\mathrm{I}^{2}$ & OR $(95 \% \mathrm{CI})$ & $\mathrm{P}$ & P-h & $\mathrm{I}^{2}$ & & & & \\
\hline Totla & 10 & $1.09(0.98,1.21)$ & 0.12 & 0.03 & 52.8 & $0.95(0.79,1.14)$ & 0.60 & 0.02 & 56.0 & & & & \\
\hline \multicolumn{14}{|l|}{ Cancer type } \\
\hline $\mathrm{HCC}$ & 3 & $1.25(0.99,1.58)$ & 0.07 & 0.09 & 58.6 & $1.06(0.86,1.31)$ & 0.58 & 0.49 & 0.0 & & & & \\
\hline $\mathrm{BC}$ & 2 & $1.02(0.84,1.25)$ & 0.84 & 0.14 & 55.2 & $0.86(0.70,1.05)$ & 0.14 & 0.48 & 0.0 & & & & \\
\hline CRC & 2 & $0.90(0.69,1.17)$ & 0.43 & 0.16 & 50.0 & $0.67(0.48,0.93)$ & 0.02 & 0.52 & 0.0 & & & & \\
\hline Other & 3 & $1.13(0.99,1.30)$ & 0.07 & 0.20 & 38.7 & $1.12(0.66,1.90)$ & 0.69 & 0.006 & 80.3 & & & & \\
\hline \multicolumn{14}{|l|}{ Ethnicity } \\
\hline Asian & 8 & $1.12(0.98,1.27)$ & 0.11 & 0.03 & 55.3 & $0.98(0.77,1.24)$ & 0.85 & 0.01 & 62.5 & & & & \\
\hline Other & 2 & $1.02(0.84,1.25)$ & 0.84 & 0.14 & 55.2 & $0.86(0.70,1.05)$ & 0.14 & 0.48 & 0.0 & & & & \\
\hline
\end{tabular}

*The number of studies included; RCC: renal cell cancer; CRC: colorectal cancer; ESCC: esophageal cancer; HCC: hepatocellular caicinoma; NPC: nasopharyngeal carcinoma; BC: breast cancer; OR: odds ratio; CI: confidence interval; P: $p$ value; $\mathrm{P}-\mathrm{h}$ : $p$ value of $\mathrm{Q}$ for heterogeneity 
A

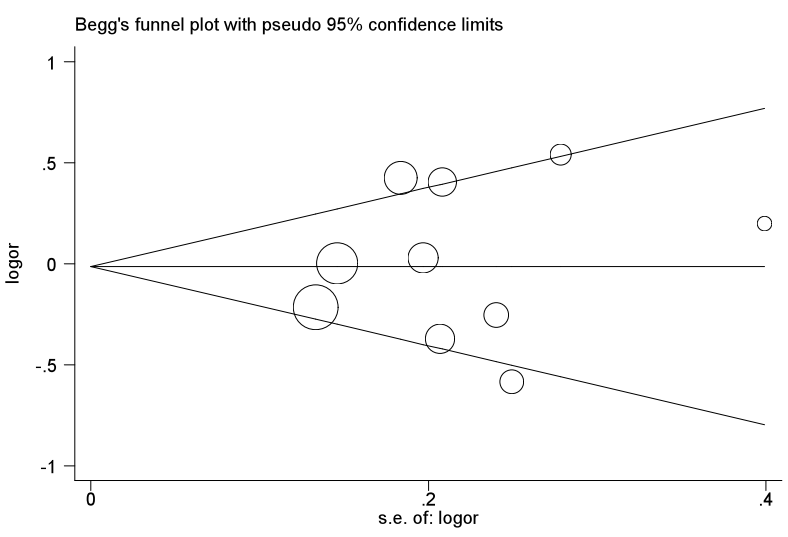

B

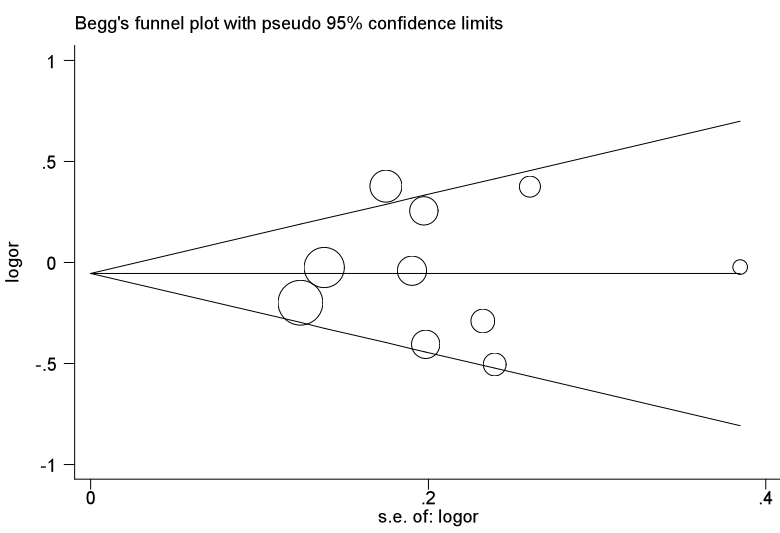

Figure 3. Begg's Funnel Plot of Publication Bias for miR-34b/c rs4938723. Each point represents a separate study for the indicated association. The natural logarithm values of odds ratio (OR) were plotted against their standard errors. (A) homozygote model; (B) recessive model.

indicated that miR-34b/c polymorphism may decreased the risk of colorectal cancer in homozygote model (CC versus $\mathrm{TT}, \mathrm{OR}=0.66,95 \% \mathrm{CI}=0.47-0.92, P=0.02)$ and in recessive model (CC versus TC+TT, $\mathrm{OR}=0.67,95 \%$ $\mathrm{CI}=0.48-0.93, P=0.02)$, but no evidence of association with hepatocellular carcinoma, breast cancer, and other cancers in any genetic models (Table 2). Figure 2 gives the forest plot that provides study-specific and pooled ORs for the association of miR-34b/c polymorphism with the risk of cancers by cancer type under homozygote model and recessive model.

\section{Sensitivity Analysis and Publication Bias}

Sensitivity analysis was carried out to evaluate the influence of each individual study on pooled OR by removing each study in turn. The results indicated that the pooled OR was not changed significantly when each individual study was removed in sequence.

Begg's funnel plot and Egger's test were also performed to evaluate the publication bias of included studies. The results indicated that no significant publication bias was detected from the Begg's funnel plot and Egger's test for all the comparison models. Figure 3 gives the Begg's funnel plot of publication bias for miR-34b/c rs4938723 under homozygote model and recessive model.

\section{Discussion}

MiR-34b/c rs4938723 is located in promoter region of pri-miR-34b/c and may generate a GATA-X binding site by bioinformatic prediction, thus the existence of rs4938723 polymorphism may affect the expression of $\mathrm{miR}-34 \mathrm{~b} / \mathrm{c}$ by transcriptional mechanisms. Evidence from studies showed that miR-34b/c can arrest cell cycle in G1 phase, the abnormal expression of miR-34b/c can affect cell proliferation and colony formation (Corney et al., 2007; Hermeking, 2010).

Several studies were conducted to investigate the association between $\mathrm{miR}-34 \mathrm{~b} / \mathrm{c}$ rs 4938723 polymorphism and risk of various cancers, including hepatocellular carcinoma, colorectal cancer, breast cancer, and other cancers. However, the results were contradictory and inconclusive. To better understanding of the association between miR-34b/c rs 4938723 polymorphism and risk of cancers, a meta-analysis with larger sample and subgroup analysis is necessary. The current study is the largest meta-analysis of the association of miR-34b/c rs 4938723 polymorphism with risk of cancers.

A total of 10 case-control studies were analyzed in this meta-analysis to perform a comprehensive evaluation of the association between $\mathrm{miR}-34 \mathrm{~b} / \mathrm{c}$ rs4938723 polymorphism and cancer risk. Our study showed that the TC genotype of rs4938723 significantly increased risk of cancers with the comparison to TT genotype when all the 10 case-control studies were merged into the metaanalysis. When stratified by ethnicity, we found the TC genotype of rs4938723 statistically significant increased risk of cancers with the comparison to TT genotype in Asian population, but not in other ethnicities. This may be due to the relatively small sample size that might affect the statistical results in other ethnicities.

In subgroup analysis by cancer type, we found significant association between miR-34b/c polymorphism and decreased risk in colorectal cancer in homozygote model and in recessive model, but not in hepatocellular carcinoma, breast cancer, and other cancers in any comparison models. This may be explained that the effect of gene polymorphism on cancer susceptibility is various to different cancer types. In addition, when all the studies were classified by cancer type, the relatively small samples in subgroup analysis might have an influence to statistical results due to insufficient statistical power.

However, some limitations should be taken into consideration in our study. First, some studies had a relatively small case-control size, and this may result in low power to statistical analysis, especially in subgroup analysis. Second, a lack of original data such as age, gender, drinking and other variables from the reviewed studies prevented an adjustment by interacting with genetic factors, could affect the evaluation of a marginal association between SNP and susceptibility to cancers. Finally, most of the patients were mainly from Asian population, this limited the application of the finding in other ethnicities from the meta-analysis.

In conclusion, this meta-analysis indicated that mir-34b/c rs4938723 polymorphism may decrease the susceptibility of colorectal cancer, but not in other cancers. 
Further studies with a larger sample size in different cancer types and additional ethnic groups are required to clarify the association of this polymorphism with cancer risk.

\section{References}

Ambros V (2004). The functions of animal microRNAs. Nature, 431, 350-5.

Bensen JT, Tse CK, Nyante SJ, et al (2013). Association of germline microRNA SNPs in pre-miRNA flanking region and breast cancer risk and survival: the Carolina Breast Cancer Study. Cancer Causes Control, 24, 1099-109.

Bossard P, Zaret KS (1998). GATA transcription factors as potentiators of gut endoderm differentiation. Development, 125, 4909-17.

Corney DC, Flesken-Nikitin A, Godwin AK, et al (2007). MicroRNA-34b and MicroRNA-34c are targets of p53 and cooperate in control of cell proliferation and adhesionindependent growth. Cancer Res, 67, 8433-8.

DerSimonian R, Kacker R (2007). Random-effects model for meta-analysis of clinical trials: an update. Contemp Clin Trials, 28, 105-14.

Egger M, Davey Smith G, Schneider M, et al (1997). Bias in meta-analysis detected by a simple, graphical test. $B m j$, 315, 629-34.

Gao LB, Li LJ, Pan XM, et al (2013). A genetic variant in the promoter region of $\mathrm{miR}-34 \mathrm{~b} / \mathrm{c}$ is associated with a reduced risk of colorectal cancer. Biol Chem, 394, 415-20.

Han Y, Pu R, Han X, et al (2013). Associations of pri-miR34b/c and pre-miR-196a2 Polymorphisms and Their Multiplicative Interactions with Hepatitis B Virus Mutations with Hepatocellular Carcinoma Risk. Plos One, 8, e58564.

Hanchard NA (2005). Genetic susceptibility and singlenucleotide polymorphisms. Semin Fetal Neonatal Med, 10, 283-9.

Hermeking $H$ (2010). The miR-34 family in cancer and apoptosis. Cell Death Differ, 17, 193-9.

Higgins JP, Thompson SG, Deeks JJ, et al (2003). Measuring inconsistency in meta-analyses. Bmj, 327, 557-60.

Jansson MD, Lund AH (2012). MicroRNA and cancer. Mol Oncol, 6, 590-610.

Ji T, Zheng ZG, Wang FM, et al (2014). Differential microRNA expression by Solexa sequencing in the sera of ovarian cancer patients. Asian Pac J Cancer Prev, 15, 1739-43.

Li L, Wu J, Sima X, et al (2013). Interactions of miR-34b/c and TP-53 polymorphisms on the risk of nasopharyngeal carcinoma. Tumor Biology, 34, 1919-23.

Mantel N, Haenszel W (1959). Statistical aspects of the analysis of data from retrospective studies of disease. J Natl Cancer Inst, 22, 719-48.

Oh J, Kim JW, Lee BE, et al (2014). Polymorphisms of the pri$\mathrm{miR}-34 \mathrm{~b} / \mathrm{c}$ promoter and TP53 codon 72 are associated with risk of colorectal cancer. Oncol Rep, 31, 995-1002.

Parlayan C, Ikeda S, Sato N, et al (2014). Association analysis of single nucleotide polymorphisms in miR-146a and miR$196 \mathrm{a} 2$ on the prevalence of cancer in elderly japanese: a case-control study. Asian Pac J Cancer Prev, 15, 2101-7.

Ruan K, Fang X, Ouyang G (2009). MicroRNAs: novel regulators in the hallmarks of human cancer. Cancer Lett, 285, 116-26.

Son MS, Jang MJ, Jeon YJ, et al (2013). Promoter polymorphisms of pri-miR-34b/c are associated with hepatocellular carcinoma. Gene, 524, 156-60.

Thakkinstian A, McElduff P, D'Este C, et al (2005). A method for meta-analysis of molecular association studies. Stat Med, 24, 1291-306.

Wigginton JE, Cutler DJ, Abecasis GR (2005). A note on exact tests of Hardy-Weinberg equilibrium. Am J Hum Genet, 76, 887-93.

Woolf B (1955). On estimating the relation between blood group and disease. Ann Hum Genet, 19, 251-3.

$\mathrm{Xu} \mathrm{Y,} \mathrm{Liu} \mathrm{L,} \mathrm{Liu} \mathrm{J,} \mathrm{et} \mathrm{al} \mathrm{(2011).} \mathrm{A} \mathrm{potentially} \mathrm{functional}$ polymorphism in the promoter region of $\mathrm{miR}-34 \mathrm{~b} / \mathrm{c}$ is associated with an increased risk for primary hepatocellular carcinoma. Int JCancer, 128, 412-7.

Yin J, Wang X, Zheng L, et al (2013). Hsa-miR-34b/c rs4938723 T>C and hsa-miR-423 rs6505162 C >A Polymorphisms Are Associated with the Risk of Esophageal Cancer in a Chinese Population. Plos One, 8, e80570.

Zhang S, Qian J, Cao Q, et al (2014). A potentially functional polymorphism in the promoter region of $\mathrm{miR}-34 \mathrm{~b} / \mathrm{c}$ is associated with renal cell cancer risk in a Chinese population. Mutagenesis, 29, 149-54. 